\title{
Zur Frage der Schichtung des Harns in der Harnblase.
}

Von

Professor Dr. G. Edlefsen in Kiel.

Im Jahre 1872 habe ich im VII. Bande dieses Archivs in einem kleinen Aufsatze „Zur Physiologie der Harnansammlung in der Blase" darauf hingewiesen, dass der Harn unter guinstigen Bedingungen sich in der menschlichen Harnblase in Schichten von verschiedener Dichtigkeit ansammelt. Meine in diesem Artikel niedergelegten Befunde haben kürzlich von zwei Seiten eine Deutung erfahren, welche von der meinigen durchaus abweicht. Ich kann es daher nicht vermeiden, auf die fragliche Angelegenheit noch einmal zurtickzukommen.

Bekanntlich habe ich in dem erwähnten Aufsatz geltend gemacht, dass eine Ansammlung des Harns in der Blase in Schichten von verschiedener Dichtigkeit bei gewöhnlicher Haltung und Lage der Blase nur zu Stande kommen könne, wenn der Gang der Absonderung ein solcher sei, dass auf anfangs abgesonderten leichten Harn immer schwerere Portionen folgen, die jenen, da sie an der tiefsten Stelle der Blase einfliessen, allmählich emporheben. Dieser Gang der Absonderung aber, so sagte ich, wird namentlich in der zweiten Hälfte der Nacht und vor allem dann stattfinden, wenn kurz vor dem Schlafengehen ein mittelgrosses Quantum Flüssigkeit, z. B. Bier, aufgenommen und wenn beim Schlafengehen um Mitternacht der um diese Zeit noch in der Blase befindliche schwerere Abendurin aus derselben entfernt wird, zu einer Zeit also, wo eben erst die dureh den Genuss des Bieres bewirkte Absonderung eines leichten Harns (die Bier-Harnfluth nach Rintaro-Mori) ihren Anfang nimmt. Dass unter solchen Bedingungen in den ersten Stunden nach Mitternacht ein sehr leichter Harn abgesondert werden muss, dem allmählich concentrirtere Portionen folgen, kann nicht zweifelhaft sein und, wenn ich nun bei der Entleerung der Blase am anderen 
Morgen die ersten Portionen am schwersten, die folgenden immer leichter fand, musste ich wohl annehmen und halte auch jetzt an dieser Annahme fest, dass die zuerst entleerten schwereren Schichten die zuletzt abges onderten waren, welche sich während der letzten Stunden des Schlafes unter den leichteren, früher abgesonderten, angesammelt hatten.

Aber auch nach geringer Flïssigkeitsaufnahme am Abend fand ich, nachdem ich gleichfalls beim Schlafengehen um $12 \mathrm{Uhr}$ die Blase entleert hatte, am anderen Morgen bei der Entleerung die erste Portion am schwersten, die folgenden leichter und habe daraus geschlossen, wie es mir auch nur natïrlich schien, dass überhaupt in der zweiten Hälfte der Nacht in der Regel anfangs noch ein verhältnissmässig leichter, gegen Morgen dagegen ein allmählich concentrirter werdender Harn abgesondert werde.

Nun aber bat Posner ${ }^{1}$ ), der in einem an sich selbst angestellten Versuch (Tabelle VI) ein mit meinen Befunden ganz übereinstimmendes Resultat erhielt, auf Grund seiner Beobachtungen an einigen anderen Versuchspersonen die Richtigkeit meiner Vorstellung von dem Gange der Harnabsonderung in der zweiten Hälfte der Nacht bestritten und behauptet in seinem Schlusssatz 4 wörtlich:

„Die von Edlefs en beobachtete Schichtung des Harns kommt nicht dadurch zu Stande, dass ein concentrirterer Morgenharn den leichteren Nachtharn in die Höhe hebt, sondern eher umgekehrt, indem leichter Morgenharndureh den sebweren Nachtharn hindurch emporsteigt".

Aehnlich scheint Rintaro Mori ${ }^{2}$ ) meine Beobachtungen zu deuten, aus denen er eine Bestätigung für die Wasserresorption aus der Blase glaubt entnehmen zu dürfen. Er sagt nämlich mit Beziehung auf dieselben: „Der Harn wird in der Blase (durch Wasserresorption) specifisch schwerer, so dass der später abgesonderte Harn bei passender Körperlage der Versuchsperson eine Schichte über dem in der Blase stagnirenden bildet." - Auch Rintaro Mori huldigt also der Anschauung, dass der leichtere, später abgesonderte, Harn in meinen Versuchen dureh die in der

1) Das Verhalten der Harnabsonderung während der Nacht. Archiv für Anatomie und Physiologie. Physiol. Abtheilung, 1887. S. 389 ff. (Heft 5).

2) Ueber die diuretische Wirkung des Bieres. Archiv für Hygiene, Bd. VII. S. 358, Anm. 6. 
Blase befindlichen schwereren Portionen emporgestiegen sei; denn er wird doch schwerlich annehmen, dass ich in der Bauch-oder Knie-ellenbogenlage geschlafen habe.

Was nun zunächst meine Beobachtungen nach reichlicher Fliissigkeitsaufnahme betrifft, so wird wohl Niemand leugnen können, dass für diese meine Deutung die richtige ist. Man würde doch der täglichen Erfahrung sämmtlicher Biertrinker entgegentreten, wollte man annehmen, dass in diesen Versuchen die leichten Harnportionen später abgesondert wären als die schweren. Ich habe überdies häufig constatirt, dass nach voraufgegangenem Biergenuss bei öfter wiederholter nächtlicher Entleerung der Blase der etwa 2-3 Stunden nach dem Schlafengehen erscheinende Harn ein sehr leichter und heller, der später entleerte bedeutend schwerer und dunkler ist. Der Schlaf bewirkt unter solchen Verbältnissen also keine Abweichung von dem gewöhnlichen Verhalten und Rintaro Mori wiurde somit die Beweiskraft seiner eigenen Versuchsergebnisse vollständig in Frage stellen, wollte er behaupten, dass bei mir die „Bierharnfluth", nachdem ich das Bier in der Zeit von 10-12 Uhr Abends getrunken hatte, erst in der Zeit von 4-7 Uhr Morgens eingetreten sei. Man braucht nur cine Anzahl meiner Tabellen den die Bier- und Weinwirkung betreffenden Tabellen von Rintaro Mori gegenüberzustellen, um zu erkennen, dass bei mir die zuletzt entleerten Portionen des Harn die zuerst abgesonderten waren.

Auch Posner ist es nicht entgangen, dass bei seinem Versuch über die Schichtung des Harns in der Blase (Tabelle VI) die Unterschiede im specifischen Gewicht der einzelnen entleerten Harnportionen „da am augenfälligsten waren, wo die Getränkzufuhr Abends die reichlichste war". Ja ich darf dreist behaupten: Posner würde in diesen Fällen noch auffallendere Unterschiede zwischen den schwersten und leichtesten Portionen gefunden haben, wenn er den Harn nicht in drei, sondern, wo es anging, wie ich, in 5-7 Gläser portionenweise entleert hätte. Wenn er z. B. in Beobachtung 5 und 6 seines Versuchs als letzte Portionen resp. 320 und $280 \mathrm{ccm}$ mit einem specifischen Gewichte von 1011 entleerte, welche anf Portionen von resp. 1019 und 1022 specifischen Gewichtes folgten, so kann es doch nicht zweifelhaft sein, dass die Gesammtmengen von 320 und $280 \mathrm{~cm}$ sich zusammengesetzt haben aus Schichten von verschiedener Dichtigkeit, die von 1018,9 und 1021,9 bis unter 1011 herabging. Denn Niemand wird glauben, 
dass die Nieren, nachdem sie eben einen Harn von 1019 abgesondert baben, unmittelbar darauf einen solchen von 1011 liefern werden, oder umgekehrt.

Wählen wir als Beispiel Posner's Beobachtung 5 (Tabelle VI) und nehmen wir einmal an, die letzt entleerte Portion von $320 \mathrm{ccm}$ mit einem specifischen Gewicht von 1011 (Fixa 8,02) sei in vier Abtheilungen von je $80 \mathrm{ccm}$ anfgefangen worden, so ergiebt die Berechnung folgendes:

Es wären entleert etwa

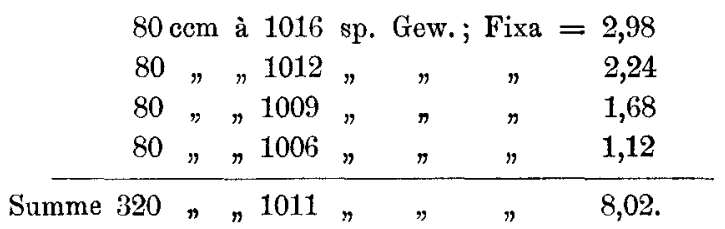

Bei weiterer Theilung würde sich wohl ergeben haben, dass die erste Portion noch schwerer, die letzte noch leichter gewesen wäre. Jedenfalls aber gehe ich nicht zu weit, wenn ich annehme, dass in diesem Versuch die oberste Harnschicht in der Blase von Posner höchstens ein specifisches Gewicht von 1006 gehabt hat.

Sicher hat Posner an den Abenden, an welchen er reichliche . Mengen Bier (1,0, 1,2 bis 2,5 Liter) genossen hatte, vor dem Schlafengehen die Blase entleert und höchst wahrscheinlich ist der um diese Zeit entleerte Harn bei ihm, wie bei anderen Menschen unter gleichen Verhältnissen, blass, vielleicht fast wasserhell, und von sehr geringem specifischen Gewicht gewesen ${ }^{1}$ ). Glaubt nun Posner wirklich, dass unmittelbar darauf in der ersten Stunde oder den ersten Stunden des Schlafs ein Harn von über 1020, ja über 1023 abgesondert worden und erst gegen Morgen wieder die Bierharnfluth zur Geltung gekommen ist, die zur Absonderung eines Harns von vielleicht nur 1002 gefibht hat? Denn das mag gleich hier bemerkt werden: Wenn Posner's Auffassung richtig, wenn der leichtere Harn durch den schwereren emporgestiegen wäre, dann müsste die unterste Harnportion, die bei der Entleerung ein specifisches Gewicht von 1023 zeigte, ursprïng-

1) Es ist zu bedauern, dass Pos s n er unterlassen hat, hierüber Bestimmungen oder Angaben zu machen, da doch die Kenntniss des Verhaltens der Harnabsonderung v o r dem Schlafengehen unbedingt erforderlich ist, wo es gilt, sich über den Verlauf derselben während des Schlafes ein Urtheil zu bilden. 
lich eine noch erheblich grössere Concentration gehabt haben und ebenso die oberste Schicht, deren Gewicht ich auf 1006 berechnete, noch erheblich leichter bei der Absonderung gewesen sein: Das geht aus meinen unten anzuführenden Versuchen über das Emporsteigen verdünnter Flüssigkeiten durch concentrirtere mit Sicherheit hervor.

Ich meine, ein solcher Gang der Absonderung in der zweiten Hälfte der Nacht ist nach vorheriger reichlicher Flïssigkeitsaufnahme physiologisch undenkbar und es liegt keine einzige Beobachtung vor, durch welche Posner die Unrichtigkeit meiner Auffassung für diese Fälle beweisen könnte. Rintaro Mori hat wahrseheinlich nur ein Referat über meine Mittheilung, deren Ort er nicht richtig angiebt, vor Augen gehabt. Wenn er sie im Orginal einsieht und meine Befunde mit seinen eigenen Beobachtungen vergleicht, wird er mir ohne Zweifel beistimmen.

Anders mag es stehen, wenn Abends wenig getrunken wurde. Dann mag es bei den nicht immer erklärlichen Schwankungen, welchen der tägliche Gang der Harnabsondernng unterworfen ist, wohl öfter vorkommen, dass in den letzten Stunden der Nacht einmal ein leichterer Harn in grösserer Menge entleert wird als in den ersten. Ich selbst habe ja auf die Möglichkeiten hingewiesen, welche zu einer vermehrten Wasserausscheidung am Ende der 24 stïndigen Tagesperiode führen können ${ }^{1}$ ). Aber Posner darf doch dass, was er in einer kleinen Zahl von Beobachtungen gefunden hat, nicht ohne Weiteres als Regel hinstellen. A priori bleibt es im Gegentheil olne Zweifel wahrscheinlicher, dass der Harn während des nächtlichen Schlafes von Stunde zu Stunde concentrirter wird und dass gerade ein abweichendes Verhalten als Ausnahme zu betrachten ist.

In Wahrbeit aber geben Po sn er's Beobachtungen wohl noch gar keinen Aufschluss über das Verhalten während eines ununterbrochenen Schlafes, weil seine Versuchspersonen mehrfach während der Nacht geweckt wurden und die Controle wohl nicht genau genug war, um sagen zu können, wie lange die Unterbrechung des Schlafes in den einzelnen Fällen gedauert hat. Ein längeres Wachen aber musste wohl, wie sich aus den, auch von Posner

1) Edlefsen, Veber das Verhältniss der Phosphorsäure zum Stickstoff im Urin. Deutsches Archiv für klin. Medicin. Bd. 29, S. 426 ff. und 459. 
citirten, Beobachtungen von Quincke ${ }^{1}$ ) ergiebt, die Harnmenge unter Abnahme ihres specifischen Gewichtes steigern und selbst eine geringere Festigkeit des Schlafes, wie sie so häufig auf wiederholte nächtliche Störungen folgt, konnte schon, wie gleichfalls aus $Q$ uincke's Beobachtungen hervorgeht ${ }^{2}$ ), genügen, um eine ähnliche Wirkung hervorzubringen. Ob auch in anderer Beziehung in Posner's Beobachtungen die Controle eine genügende war: ob es ganz ausgeschlossen ist, dass die Versuchspersonen, wenn sie geweckt wurden, getrunken haben, kann ich natürlich nicht entscheiden. Aber, wenn ich sehe, dass in einzelnen Versuchen in der Zeit von 2-7, resp. 4-7 Uhr stïndliche Mengen von $150 \mathrm{ccm}$ und darüber ausgeschieden wurden, so kann ich mich der Vermuthung nicht ganz erwehren, dass hier eine Flüssigkeitsanfnahme während der Nacht stattgefunden babe. Falls aber diese Möglichkeit sicher ausgeschlossen ist, bleibt nach den Beobachtungen von $Q$ u incke nur die Annahme ubrig, dass die Versuchspersonen in diesen Fällen nach dem letzten Wecken nicht wieder eingeschlafen sind.

Ueber den normalen Gang der Harnabsonderung in der zweiten Hälfte der Nacht unter physiologis chen Verhältnisse $\mathbf{n}$, d. h. während eines ununterbrochenen Schlafes geben also die Beobachtungen von Posner noch keinen genigenden Aufschluss. Dazu sind exactere Untersuchungen oder mindestens genauere Angaben der Versuchsbedingungen erforderlich. Vor Allem muss festgestellt werden, ob das einfache Wecken, $d$. $h$. eine ganz kurze Unterbrechung des Schlafes bei Personen, die leicht wieder einschlafen, gentugt, um bei Ausschluss jeder neuen Flüssigkeitsaufnahme eine Aenderung der Harnabsonderung im Sinne der Quincke'schen morgentlichen Harnfluth zu bewirken. Sollte sich dies, wie ich freilich nicht glaube, bestätigen, so würde man ja uiber den Gang der Harnabsonderung w äh re nd des Se h lafes iiberhaupt nur durch die. Untersuchung der in der Blase angesammelten Schichten des Harns Auskunft erhalten können. Aber freilich, dann ist es auch von entscheidender Bedeutung, genau zu wissen, wie diese Schichtung zu Stande kommt.

1) Ueber den Einfluss des Schlafes auf die Harnabsonderung. Archiv für experiment. Pathologie und Pharmakologie. Bd. VII, S. 115 ff.

2) Ebenda S. 122 und 123. 
Bis zum Erscheinen der Posner'schen Arbeit habe ich geglaubt, dass dieselbe nur möglich sei, wenn immer concentrirter werdende Flüssigkeit mit nicht zu grosser Einflussgeschwindigkeit am Boden des Gefässes eintritt und die darin bereits befindliche leichtere emporhebt, und habe, wie erwähnt, diese Vorstellung auch auf die Harnansammlung in der Blase übertragen. Posner sagt im Gegentheil: man muss annehmen, dass der leichte Morgenharn durch den schwereren Nachtharn hindurch in die Höhe steigt, und meint weiter, dass dieses Verbalten physikalisch gewiss ebenso denkbar sei, wie das Emporheben leichter Fliussigkeitsschichten durch schwerere.

Dass es denkbar oder vielmehr dass es möglich ist, das gebe ich jetzt, nachdem ich mich durch eigene Versuche (s. u.) davon überzeugt habe, vollkommen zu; aber ,ebenso denkbar" ist es sicher nicht. Denn schon a priori kann man sagen, dass ein Hindurchtreten verdünnter wässeriger Lösungen durch concentrirtere ohne eine s of or t eintretende, wenigstens theilweise, Ausgleichung ihres Gehalts an festen Bestandtheilen gar nicht möglich ist, während bei dem Emporheben einer leichten Flüssigkeit durch eine schwerere eine solche Ausgleichung doch nur an der Bertihrungschicht geschehen kann und bei Vermeidung des Schüttelns immer nur allmählich stattfinden wird. Das Experiment beweist die Richtigkeit dieser Annahme.

Posner ist freilich der experimentelle Nachweis fur seine Auffassung „bisher noch nicht geglückt". Weshalb nicht? Es war doch so leicht, diesen Nachweis zu führen. Aber war Posner denn, so lange er ihn nicht geführt hatte, berechtigt, meine wohlbegründete Anschauung einfach uiber den Haufen za werfen und in seinem Schlusssatz positiv zu sagen: Die von Edlefsen beobachtete Schichtung .... kommt nicht dadurch zu Stande etc.? Hätte er „nicht immer" oder ,nicht nothwendig" gesagt, so könnte ich die Berechtigung dieser Einschränkung nicht bestreiten, da die Schichtung von ungleich schweren Flüssigkeiten, wie ich durch sehr einfache Versuche festgestellt habe, wirklich in der Weise zu Stande kommen kann, wie Posner vermuthet. Aber, wie wir gleich sehen werden, bleibt es auch nach dem Ergebniss dieser Versuche wahrseheinlich, dass da, wo man erhebliche Unterschiede in dem specifischen Gewichte der einzelnen entleerten Harnportionen findet, die Schichtung in der Weise vor sich gegangen 
ist, wie $\mathrm{i}$ c h angenommen habe. Es kann nämlich allerdings, wie ich fand, eine specifisch leichtere Flüssigkeit durch Flüssigkeitsschichten von grösserer Dichtigkeit emporsteigen und sicb über diesen sammeln. Aber, selbst bei der vorsichtigsten Anordnung des Versuchs, d. h. bei möglichster Verminderung des Drucks und der Geschwindigkeit, mit welcher sie durch eine feine Oeffnung einströmt, findet doch eine stärkere Ausgleichung des specifischen Gewichts der einzelnen Schichten statt, als bei der umgekehrten Anordnung, d. h. bei der Emporhebung leichterer Flïssigkeit durch schwerere, und diese Ausgleichung tritt um so vollständiger ein, je geringer der Unterschied in der Schwere der einzelnen Flïssigkeitsmengen ist und je rascher das Einströmen gescbieht.

Ueber di e S c bi c htung vonschwererer Flüs sigkeit unter leichteren Portionen besitze ich noch zwei Versuchsprotocolle aus dem Jahr 1872. Dieselben mögen hier zunächst folgen.

Ich benutzte bei diesen Versuchen als Reservoir eine Schusterkugel, welche genau $1520 \mathrm{ccm}$ fasste, und als Füllflüssigkeit Kochsalzlösung verschiedener Concentration, von welcher einzelne Portionen zur leichteren Unterscheidung verschieden gefärbt waren. Um das Einströmen der Flüssigkeit möglichst langsam geschehen zu lassen, liess ich sie durch ein Filter gehen. An dem Glastrichter war mittels eines kurzen Schlauches ein zugespitztes Glasrohr befestigt, dessen feine Oeffnung sich dicht über dem Boden des Gefässes befand. Durch meine ärztliche Thätigkeit wurde das Nachgiessen der Flüssigkeit auf das Filter oft unterbrochen, so dass in beiden Versuchen fast 32 Stunden bis zur vollständigen Füllung der Kugel vergingen und die einzelnen Flüssigkeitsschichten demnach lange Zeit mit einander in Berührung blieben. Während der Füllung blieben die einzelnen Portionen, wie die Färbung erkenneu liess, zunächst scharf von einander getrennt. Erst gegen das Eade des Versuchs machte sich eine leichte Verwaschung der Grenzen farbloser Schichten gegen die darüber oder darunter stehenden gefärbten bemerkbar. Die Entleerung wurde mittels eines Hebers vorgenommen, indem zunächst die unterste Portion entfernt wurde, welcher dann die darüber stehenden Schichtẹn der Reihe nach folgten Die Tabellon habe ich so geordnet, dass der zuletzt eingelaufenen (schwersten) Portion die zuerst entleerte gegenübersteht u. s. w. In Versuch 2 ging der Kntleerung eine mehrmalige vollständige Umkehrung der Kugel vorauf, wobei die letztere sich. um die Flüssigkeit herum verschob, während diese in Ruhe verharrte und höchstens eine ganz leichte Vermischung der Flüssigkeitsschichten an den Grenzen bemerken liess. 
Versuch 1 .

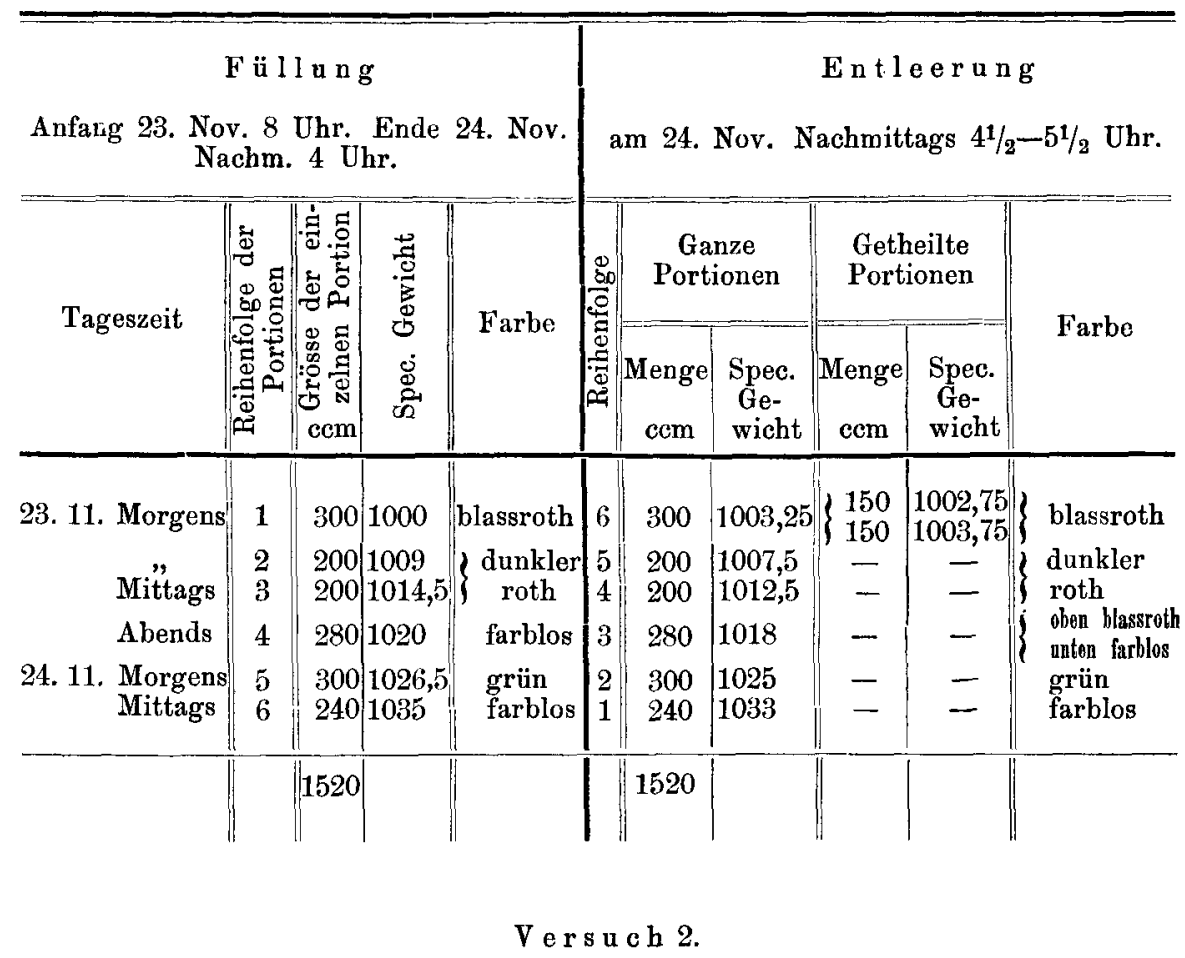

F üllung vom 30. 11. M. $8 \mathrm{Uhr}-1.12$. Entleerung am 2. 12. Abends Nm. 4 Uhr. 10-11 Uhr.

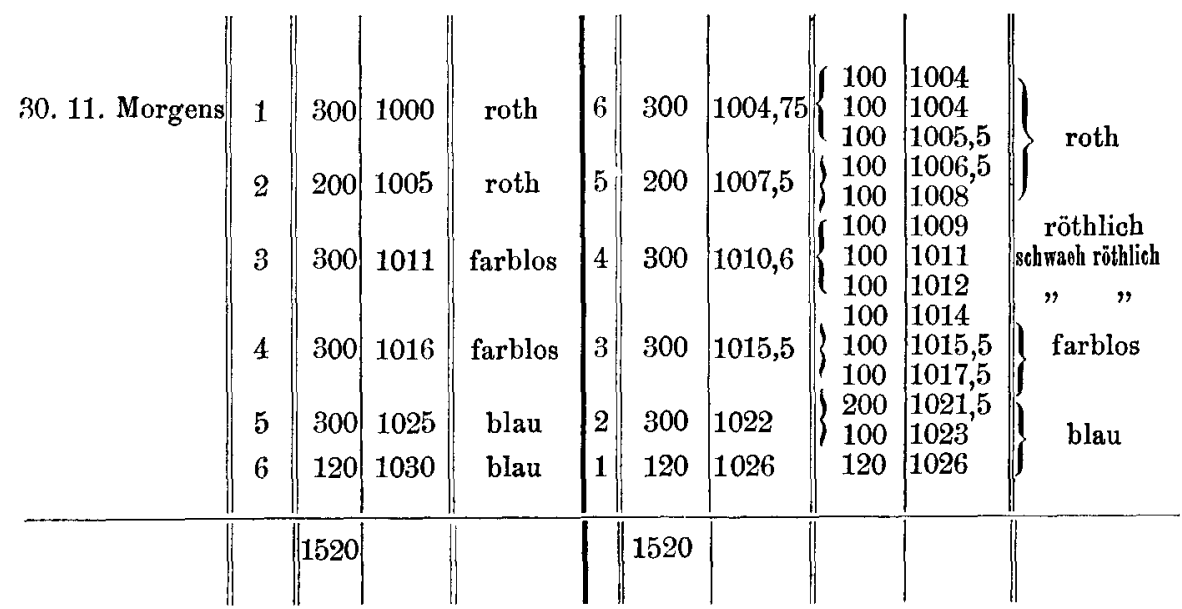


$\mathrm{Da}$ in den vorstehenden beiden Versuchen die Bedingungen vielleicht im Vergleich mit der normalen Ansammlung des Harns in der Blase als allzu günstig gewählt erscheinen könuten, so habe ich neuerdings noch folgenden Versuch angestellt, bei welchem die Bedingungen absichtlich ungünstig gestaltet wurden, indem das Finfliessen der einzelnen Portionen ohne Filter und, zwar meistens in continuirlichem, langsamem Strom, aber doch zuweilen, namentlich Anfangs, auch durch nicht vorhergesehenes rascheres Ausfliessen aus dem Ausgussgefäss stossweisse erfolgte, in welchem Falle dann meistens auch noch Luftblasen mitgerissen wurden. Trotz dieser ungünstigen Verhältnisse sind, wie man sieht, die Unterschiede im specifischen Gewichte der einzelnen Portionen sehr bedeutende geblieben, wenn auch in den zuerst eingeflossenen leichteren Schichten die Ausgleichung etwas beträchtlicher ausgefallen ist als in den vorigen Versuchen. Als Füllgefäss wurde eine Kolbenflasche mit fast planer Grundfläche benutzt. Die Mündung des zugespitzten Glasrohrs, welches mit dem Trichter verbunden war, befand sich einige Millimeter oberhalb des Bodens der Flasche. Dadurch musste eine Mischung der einströmenden mit der bereits eingeflossenen Flüssigkeit noch begünstigt werden. Daraus erklärt es sich auch, dass bei der Entleerung die letzte, nicht mehr durch Heber sondern durch Ausgiessen entfernte, Portion ein höheres specifisches Gewicht zeigte als die fünfte und sechste, welche ebenso wie die vorigen mittels Hebers berausgehoben wurden.

Versuch 3 .

28. März 1888.

\begin{tabular}{|c|c|c|c|c|c|c|c|c|}
\hline \multicolumn{5}{|c|}{ F ü 11 ung } & \multicolumn{4}{|c|}{ Entleerung } \\
\hline 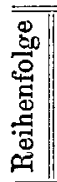 & $\begin{array}{c}\text { Menge } \\
\text { ccm }\end{array}$ & 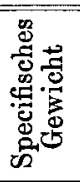 & Farbe & 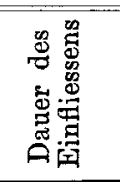 & 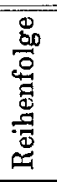 & $\begin{array}{c}\text { Menge } \\
\text { cem }\end{array}$ & 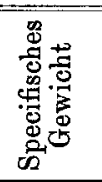 & Farbe \\
\hline \begin{tabular}{l||}
1 \\
2 \\
3 \\
4 \\
5 \\
6 \\
7
\end{tabular} & $\begin{array}{l}100 \\
100 \\
100 \\
100 \\
100 \\
100 \\
100\end{array}$ & $\begin{array}{l}1000 \\
1005 \\
1010 \\
1015 \\
1020 \\
1025 \\
1030\end{array}$ & $\begin{array}{c}\text { farblos } \\
\text { roth } \\
\text { farblos } \\
\text { violett } \\
\text { farblos } \\
n\end{array}$ & $\begin{array}{rc}0 & \text { Min. } \\
4 & \text { " } \\
7 & " \\
8 & " \\
8 & \prime \\
10 & \prime \\
8 & ,\end{array}$ & $\begin{array}{l}7 \\
6 \\
5 \\
4 \\
3 \\
2 \\
1\end{array}$ & $\begin{array}{l}100 \\
100 \\
100 \\
100 \\
100 \\
100 \\
100\end{array}$ & $\begin{array}{l}1007 \\
1003,5 \\
1006 \\
1016 \\
1018 \\
1023,5 \\
1027 \\
\end{array}$ & $\begin{array}{l}\text { dunkel roth } \\
\text { hell roth" } \\
\text { hell violett } \\
\text { dunkel violett } \\
\text { hell " } \\
\text { " }\end{array}$ \\
\hline & 700 & 1015 & & & & 700 & 1014,5 & . \\
\hline
\end{tabular}

Die folgenden Versuche beziehen sich auf das Verhalten beim Emporsteigen leichterer Flussigkeit durch schwerere. 
Die Anordnung ist bei jedem einzelnen Versuch angegeben. Ich beschränke mich auf die Mittheilung einer kleinen Zahl von Versuchen, deren jeder eine besondere Modification repräsentirt, indem theils das Verhalten bei grösserer Verschiedenheit des specifischen Gewichts einmal (Versuch 4 und 6) bei langsamem, dann (Versuch 5) bei raschem Einströmen, theils das Verhalten bei geringen Unterschieden der specifisehen Gewichte bei langsamem Einfliessen beobachtet wurde (Versuch 7 und 8). Noch mehr derartige Versuche anzuführen, hielt ich für überflüssig. Ich darf es Posner uberlassen, wenn er es für wünschenswerth hält, die Zahl der Versuche zu vermehren.

\section{V e rs u ch 4}

20. Decbr. 1887. In ein cylindrisches Gefäss werden durch ein kleines Filter $90 \mathrm{ccm}$ Kochsalzlösung von 1015 specifischem Gewicht eingeführt. Die filtrirende Flüssigkeit fliesst durch ein zugespitztes bis auf den Boden des Gefässes reichendes Glasrohr langsam ein in der '/eit von $93 / 4-10$ Uhr Abends. Von $10 \mathrm{Uhr}$ an wird auf dasselbe kleine liilter eine mit luchsin gefärbte Kochsalzlösung von 1001,5 specifischem Gewicht gebracht. Im Lauf von 40 Minuten fliessen $90 \mathrm{ccm}$ dieser Lösung durch. Anfangs sieht man deutlich neben dem Glasrohr rothe Flüssigkeitsströme nach oben steigen und über der ungefärbten Kochsalzlösung eine Schicht rother Flïssigkeit sich ansammeln. Allmählich färbt sich jedoch die ganze Flüssigkcit roth; nur scheint die obere Schicht etwas dunkler gefärbt zu bleiben. $10 \mathrm{Uhr} 45$ Minuten Entleorung mittels Hebers:

Unterste Portion $90 \mathrm{~cm}$, spec. Gew. 1009,5.

Obere Portion $90, \quad, \quad, 1007$.

Versuch 5 .

21. Decbr. 1887. $125 \mathrm{ccm}$ mit Fuchsin gefärbter Kochsalzlősung von 1010,5 specifischem Gewicht werden in ein cylindrisches Gefäss gebracht, darauf, durch einen Trichter mit zugespitztem, bis auf den Boden des Gefässes reichendem Glassrohr $100 \mathrm{ccm}$ ungefärbtes Leitungswasser ohne Filter zugelassen, welche in kaum 5 Minuten einfliessen. Während des Einfliessens ist wohl ein aufsteigender Flüssigkeitsstrom, aber keine Trennung in ungleich gefärbte Schichten zu bemerken. Sofortige Enticerung mittels Hebers:

Unterste Portion $125 \mathrm{ccm}$, spec. Gew. 1005.

OberePortion $100, \quad, \quad, 1005$.

Versuch 6.

25. Decbr. 1887. In eine Kolbenflasche werden $100 \mathrm{ccm}$ Kochsalzlösung von 1020 specifischem Gewicht gebracht. Durch ein Filter fliessen sodann 
in 10 Minuten $100 \mathrm{ccm}$ Kochsalzlösung ron 1010 specifischem Gewicht am Boden des Gefässes ein, darauf in 20 Minuten $140 \mathrm{ccm}$ intensiv gefärbte Fuchsinlösung von eben üher 1000 specifischem Gewicht. Aufsteigende rothe Flüssigkeitsströme wie in Versuch 4. Darauf sofortige Entleerung mittels Hebers, die in weniger als 10 Minuten beendigt ist:

Un terste $100 \mathrm{cem}$, ziemlich stark roth gefärbt: spec. Gewicht $=1013$.

Mittlere 100, , etwas intensiver gefärbt: $\quad, \quad, \quad 1010$.

Oberste 140 "dunkler roth gefärbt: $\quad, \quad, \quad 1005$.

Versuch 7 .

2. Januar 1888. In eine Kolbenflasche fliessen durch ein Filter langsam am Boden des Gefässes der Reihe nach je $50 \mathrm{ccm}$ einer Kochsalzlösung von resp. 1005,5, 1004,5, 1004, 1003, 1002 und 1001 specifischem Gewicht ein. Bei der sodann vorgenommenen Entleerung mittels Hebers zeigte jede Portion ein specifisches Gewicht von 1003 mit Ausnahme der letzten, die nur 1002,5 wog.

\section{Versuch 8.}

29. März 1888.

Als Füllgefäss wurde eine Schusterkugel benutat. Das Einfliessen geschah langsam durch ein Filter. Das zugespitzte Einflussrohr reichte genau bis an die tiefste Stelle der Kugel. Bei dem Einfliessen der violett gefärbten Portion sah man wie die im Apparat bereits befindliche Flüssigkeit sich gleichmässig blass violett färbte, während eine dunkler violette Schicht sich darüber lagerte, welche auch nach dem späteren Einfliessen der leichteren Portionen von den benachbarten (unteren wie oberen) Schichten deutlich, wenn auch nicht scharf getrennt blieb.

\begin{tabular}{|c|c|c|c|c|c|c|c|c|}
\hline \multicolumn{5}{|c|}{ F ü $11 \mathrm{u} n g$} & \multicolumn{4}{|c|}{ Entle erung } \\
\hline 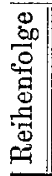 & $\begin{array}{c}\text { Menge } \\
\text { ccm }\end{array}$ & 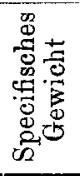 & Farbe & 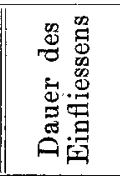 & 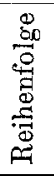 & $\begin{array}{c}\text { Menge } \\
\mathrm{ccm}\end{array}$ & 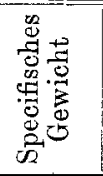 & Farbe \\
\hline \begin{tabular}{l|}
1 \\
2 \\
3 \\
4 \\
5 \\
6
\end{tabular} & $\begin{array}{l}100 \\
100 \\
100 \\
100 \\
100 \\
100\end{array}$ & $\begin{array}{l}1030 \\
1025 \\
1020 \\
1015 \\
1011 \\
1006\end{array}$ & $\begin{array}{c}\text { farblos } \\
\text { violett } \\
\text { farblos } \\
", \\
",\end{array}$ & $\begin{array}{cc}0 & \text { Min. } \\
10 & n \\
25 & " \\
10 & " \\
10 & " \\
20 & "\end{array}$ & $\begin{array}{l}1 \\
2 \\
3 \\
4 \\
5 \\
6\end{array}$ & $\begin{array}{l}100 \\
100 \\
100 \\
100 \\
100 \\
100\end{array}$ & $\begin{array}{l}1020 \\
1019,5 \\
1018,5 \\
1017,5 \\
1016,5 \\
1015\end{array}$ & $\begin{array}{l}\text { fast farblos } \\
\text { " } " \\
\text { hell violett } \\
\text { dunkler violett } \\
\text { blass violett }\end{array}$ \\
\hline & 600 & 1018 & & 75 Min. & & 600 & 1018 & \\
\hline
\end{tabular}


Aus diesen Versuchen ergiebt sich, wie oben bereits kurz angedeutet wurde, dass eine Schichtung von Flüssigkeiten verschiedener Concentration über einander möglich ist, auch wenn die leichtere durch die schwerere emporsteigen muss, dass aber doch eine Mischung der einzelnen Flüssigkeitsschichten viel leichter vermieden wird, wenn die leichte Flïssigkeit durch die schwere emporgehoben wird. Es ergiebt sich daraus ferner, dass be i geringer Verschiedenheit der Concentration der einzelnen Flüssigkeitsportionen aueh bei ganzlangsamem Einströmenderselben eine Ausgleichung mit dem vorhandenen Inhalt des Gefässessehr leichteintritt, sobald dieleichtere Flissigkeit dureh die sehwerere emporsteigt, dagegenausbleibt, wenn dieschweredieleichtere Schicht emporhebt. Weit leichter noch tritt im ersteren Falle die Mischung und eine vollständige Ausgleichung des specifischen Gewichts be i ras chem Einström en der F I ü ssigkeit ein, ein Moment, welches bei der Harnansammlung in der Blase wohl in's Gewicht fällt, da das Einströmen hier in Folge der peristaltischen Bewegung der Ureteren doch wohl häufig stossweis und mit verhältnissmässig grosser Geschwindigkeit vor sich gehen dürfte. Dagegen geht aus dem Versuch 3 klar hervor, d a s s a u h rascheres und absatzweises Einströmen dieschichtung nichtverhindert, wenndieschwereren Portionen auf dieleichteren folgen und diese e m por heben.

Wenn ich nun die grossen Unterschiede im specifischen Gewicht in Betracht ziehe, welche die einzelnen $\mathrm{Harnporti}$ on e $n$ in meinen und z. T. auch in Posner's Beobachtungen $\mathrm{nach}$ reich lich er Flüs sigkeits a u f a h m e zeigten, so meine ich, dass man daraus mit Sicherheit auf die Richtigkeit meiner fruber gegebenen Erklärung schliessen kann, und dass, wo so prägnante Unterschiede beobachtet werden, überhaupt der Schluss berechtigt ist, dass die Füllung der Blase unter a ll mä h l i ch z u n e h mender Con centration des einfliessenden Harns vor sich gegangen sei. Denn es kann doch wohl nicht zweifelhaft sein, dass die Zu- oder Abnahme des specifischen Gewichts des Nierensecretes nur i n ganzallmählichen U ebergängen 
erfolgt ${ }^{1}$ ). Eine sprungweise Aenderung, ein $\mathrm{pl} \ddot{\mathrm{l}} \mathrm{t} \mathrm{z} \mathrm{l}$ i c h e r Wechsel zwischen der Absonderung eines leichten und eines schweren Harns oder umgekehrt scheint mir physiologisch undenkbar zu sein. Bei dem von Posner angenommenen Vorgang der Schichtung kommen aber grössere Unterschiede im specifischen Gewicht der Schichten nur dann zur Beobachtung, wenn die Concentration der einzelnen einfliessenden Flüssigkeitsportionen eine weit anseinanderliegende ist, wie in Versuch 4 und 6.

Wo die Unterschiede gering sind wie in meinen Beobachtungen nach geringer Flüssigkeitsaufnahme während der Nacht oder bei aufrechter Haltung am T a ge (a. a. 0. Beobachtung 11-20), da gebe ich zu, dass es sich nicht ganz sicher wird entscheiden lassen, unter welcher Reihenfolge der Absonderung die Schichtung zu Stande gekommen ist. Bei den geringen Unterschieden im specifischen Gewichte der einzelnen Portionen, welehe in diesen Versuchen beobachtet wurden, könnte ich wohl zu Gunsten meiner Auffassung geltend machen, dass die Bedingungen für die Schichtung mittelst Emporsteigens leichterer Flüssigkeit durch schwerere entsprechend meinen Versuchsergebnissen (s. o.) keine günstige waren. Aber auf der anderen Seite kann ich den Einwand nicht entkräften, dass diese Unterschiede ursprïnglich beim Eintritt des Harns in die Blase grösser gewesen und erst in Folge des Durchtretens der leichteren Flüssigkeit durch die schwereren Schichten bis za dem beobachteten Grade abgeschwächt sein können.

Im Ganzen aber spricht doch auch hier, wie ich meine, die grössere Wahrscheinlichkeit für meine ursprüngliche Auffassung: Meine Thätigkeit wähiend der Ansammlung des Harns in der Blase in den Vormittagsstunden war gewiss nicht der Art, dass sie das Zustandekommen der Schichtung begïnstigte, und die häufige Erschütterung des Blaseninhalts beim Gehen, Treppensteigen, Büicken u. s. w. musste während des Durchtritts

1) Dieser allmähliche Uebergang würde natürlich, wie ich schon 1872 hervorhob (a. a. O. S. 507), auch bei der Entleerung des Harns deutlich erkennbar werden, wenn man denselben in kleineren Portionen - etwa von je $10 \mathrm{ccm}$ - auffinge und das spec. Gewicht derselben piknometrisch bestimmte. Zur Ausführung dieses Versuches hat es mir bisher an Zeit und Gelegenheit gefehlt.

E. Pfüger, Archiv f. Physiologie. Bd. XIIII. 
leichter Flüssigkeit durch concentrirte Sehichten jedenfalls die Mischung sehr begünstigen. Wenn ich trotzdem nach 5-6stïndiger ärztlicher Thätigkeit noch Unterschiede im specifischen Gewicht der einzelnen gesebichteten Harnportionen von 1014,5-1016, von $1007-1010$, von $1018-1020$ u. s. w eonstatiren konnte ${ }^{1}$ ), so dürfte das wohl mehr für den umgekehrten Gang der Harnabsonderung sprechen.

Bei meinen Beobachtungen über die Schichtung des Harns in der Blase während der Nachtruhe nacb geringer Flüssigkeitsau fnahme scheint mir der Umstand denselben Hergang wahrscheinlich zu machen, dass trotz der geringen Harnmengen, welche sich jedesmal in der Blase fanden, doch so deutliche Unterschiede im specifischen Gewicht der einzelnen Schichten hervortraten, dass z. B. bei einer Menge von $181 \mathrm{ecm}$ das specifische Gewicht der 3 anfgefangenen Portionen 1022, 1026 und 1028 betrug, in einem anderen Versuch $182 \mathrm{ccm}$ sich in 2 Portionen von 1022 und 1025 theilten u. s. w. ${ }^{2}$ ).

Aber, wie bemerkt, eine vollkommen sichere Entscheidung ist hier nicht möglich und es muss abgewartet werden, ob directe, mit aller Vorsicht angestellte Beobachtungen uber den Gang der Harnabsonderung in der zweiten Hälfte der Nacht, zu deren Ausführung ich bereits Anregung gegeben habe, uns den erwünschten Aufschluss verschaffen werden.

Schliesslich erlaube ich mir noch mit wenigen Worten auf die Frage der Wasserresorption aus der Harnblase einzugehen. Der Gedanke, meine Beobachtungen über die Schichtung des Harns in der Blase in dieser Richtung za verwerthen, hat mir schon 1872 nahe gelegen; aber ich habe es damals mit voller Ueberlegung unterlassen, auch nur ein Wort darüber zu sagen, weil mir die Angelegenheit durch die kurz vorher erschienenen Untersuchungen von $\mathrm{Treskin}^{3}$ ) erledigt zu sein schien. Wenn ich jetzt darauf zurückkomme, so geschieht es nur, weil Rintaro Mori (a. a. O.) geglaubt hat, meine Beobachtungen zu Gunsten seiner Theorie von der Wasserresorption aus der Blase in's Feld führen zu dürfen.

1) Beobachtung 16, 18 und 19.

2) Beobachtung 11 und 13.

3) Beiträge zur Physiologie der Harnblase und der Nieren. Dieses Archiv, Bd. V, S. 324 ff. 
Ich bemerke nur kurz, dass meine Versuche, w e n n man daraus in Bezug auf diese Frage Schltisse ziehen will, wohl nur das Gegentheil von Rintaro Mori's Ansicht beweisen können. Denn, wenn die obersten Schichten des Harns in der Blase während einer siebenstiundigen Retention in derselben noch in einer Menge von $125-260 \mathrm{ccm}$ ein specifisches Gewicht von 1001,51002 behaupten können (Beobachtung 2 und 5), so dürfte das doch wohl ge ge n die Annahme einer Wasserresorption aus der Blase sprechen. Näher auf diese Frage einzugehen, scheint mir an diesem Orte nicht erlanbt, da ich keine weiteren eigenen Beobachtungen anzuführen habe. Nur die eine kurze Bemerkung kann ich mir nicht versagen, dass Rintar o Mori das Gewicht der Untersuchungen von Treskin, welehe im Gegentheil den Uebergang von Wasser aus Blut und Lymphe in den Blaseninhalt bewiesen, doch wohl etwas unterschätzt hat. Dass die absolute Zunahme des Kochsalzgehalts des Harns nicht gegen seine Annahme spreche, bat Treskin selbst ausdriicklich hervorgehoben.

\section{Ueber die von $\nabla$. Kries wider die Theorie der Gegenfarben erhobenen Einwände.}

\section{Mittheilung.}

Ueber die sogenannten Ermü dungserscheinungen.

Von

\section{Ewala Hering,}

Prof. der Physiologie an der deutschen Universität Prag.

Kries hat auffallenderweise bei seiner Erörterung der Contrast- und Ermiudungserscheinungen einen der wesentlichsten Unterschiede zwischen meiner und der Dreifarbentheorie völlig unberück. sichtigt gelassen. Nach meiner Theorie sind die Erregbarkeitsänderungen and also auch die "Ermüdungen" des Sehorgans 